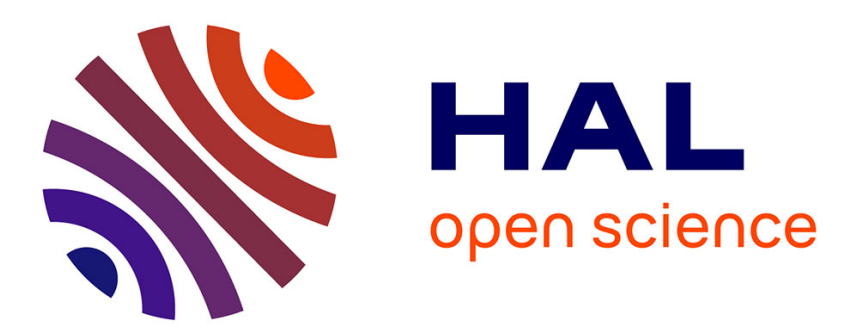

\title{
Simple pre-distortion schemes for improving the power efficiency of SOA-based IR-UWB over fiber systems
}

H Taki, S Azou, A Hamie, Ali Al Housseini, A Alaeddine, A Sharaiha

\section{To cite this version:}

H Taki, S Azou, A Hamie, Ali Al Housseini, A Alaeddine, et al.. Simple pre-distortion schemes for improving the power efficiency of SOA-based IR-UWB over fiber systems. Optics Communications, 2017, 10.1016/j.optcom.2016.07.066 . hal-01353980

\author{
HAL Id: hal-01353980 \\ https://hal.science/hal-01353980
}

Submitted on 17 Aug 2016

HAL is a multi-disciplinary open access archive for the deposit and dissemination of scientific research documents, whether they are published or not. The documents may come from teaching and research institutions in France or abroad, or from public or private research centers.
L'archive ouverte pluridisciplinaire HAL, est destinée au dépôt et à la diffusion de documents scientifiques de niveau recherche, publiés ou non, émanant des établissements d'enseignement et de recherche français ou étrangers, des laboratoires publics ou privés. 


\title{
Simple Pre-distortion Schemes for Improving the Power Efficiency of SOA-Based IR-UWB over Fiber Systems
}

\author{
H. Taki*†, S. Azou ${ }^{\dagger}$, A. Hamie ${ }^{* \ddagger}$, A. Al Housseini*, A. Alaeddine* and A. Sharaiha ${ }^{\dagger}$ \\ * Lebanese University, Faculty of Science I, LPE research center, Beirut, Lebanon \\ $\dagger$ ENIB / CNRS UMR 6285 Lab-STICC, Brest, France \\ $\ddagger$ CRITC research center, Arts Sciences and Technology University in Lebanon (AUL)
}

\begin{abstract}
In this paper, we investigate the usage of SOA for reach extension of an impulse radio over fiber system. Operating in the saturated regime translates into strong nonlinearities and spectral distortions, which drops the power efficiency of the propagated pulses. After studying the SOA response versus operating conditions, we have enhanced the system performance by applying simple analog pre-distortion schemes for various derivatives of the Gaussian pulse and their combination. A novel pulse shape has also been designed by linearly combining three basic Gaussian pulses, offering a very good spectral efficiency $(>55 \%)$ for a high power $(0 \mathrm{dBm})$ at the amplifier input. Furthermore, the potential of our technique has been examined considering a 1.5 Gbps-OOK and 0.75 Gbps-PPM modulation schemes. Pre-distortion proved an advantage for a large extension of optical link $(150 \mathrm{Km})$, with an inline amplification via SOA at $40 \mathrm{Km}$.
\end{abstract}

\section{INTRODUCTION}

UWB is known to be a promising technology for shortrange broadband wireless communications, thanks to its many interesting features including high data rate, immunity to multipath propagation, flexibility in reconfiguring data rate and power, very good time domain resolution, accurate mobile user location, and easy data protection [1] [2]. Hence, UWB is a good candidate for various applications like local and wide area networks, sensor networks, emergency communications, radar, remote sensing, and military applications [3]. In 2002, the Federal Communication Commission (FCC) endorsed UWB devices to use the unlicensed frequency band [3.1,10.6] $\mathrm{GHz}$, where the maximum emitted power must not exceed $41.3 \mathrm{dBm} / \mathrm{MHz}$, in order to avoid interference with existing communication systems [4]. Impulse Radio (IR), consisting in transmitting ultra short duration pulses in baseband, is an attractive UWB technology due to its low cost and complexity [5], [6]. Extending the coverage areas of UWB systems using optical fiber technology, is a pertinent approach that has been demonstrated in recent studies [7]-[11]. UWB transmission over fiber requires direct optical pulse generation [12], [13] or electro-optical conversion via intensity modulators [14]. Inline amplification is a key function for achieving the reach extension of any optical communication network. As low cost devices, Semiconductor Optical Amplifiers (SOAs) [15] may be good candidates in the context of IR over fiber transmissions, the essence of IR being the simplicity with the objective of a wide use. Unfortunately, a main drawback for this approach is the non-linear effects inherent in the amplification, causing strong distortions in the propagating signals and a degradation in the power efficiency. Due to the regulation constraints associated to UWB free space propagation, highly efficient transmission is required which means that the pulse shapes must be preserved along the transmission in order to collect the maximum possible power under the FCC spectral mask. Hence, overcoming SOA non-linear distortions becomes a key objective. In the literature, numerous linearization techniques dealing with over fiber transmission have been investigated [16]-[18]. Here, we examine the potential of analog predistortion against SOA non-linearity; by tuning some key parameters of pulses belonging to the Gaussian family or of their combinations [19], we have succeeded in designing highly efficient pulse shapes at the receiver side (photodetector output). The best parameters of pre-distorted input pulses were extracted via global optimization based on the interior point algorithm. The resultant waveforms have been examined considering modulated IR signals; it is shown that a significant enhancement in the efficiency can be achieved for a large optical link extension, while operating at limited cost and complexity.

\section{IR-UWB OVER FIBER SYSTEM ARCHITECTURE AND SOA IMPACT ON OUTPUT PULSES RESPONSE}

The Radio-over-Fiber ( $\mathrm{RoF}$ ) utilized is depicted in fig. 1. The transmitter relies on a Mach-Zehnder Modulator (MZM) with half-wave voltage $V \pi$ of $6 \mathrm{~V}$, biased at $V_{D C}=1.5 V_{\pi}$ by the electrical IR-UWB signal (with a peak-to-peak voltage $V_{p p}$ ), a continuous wave light being applied at its input (laser diode signal). The channel is made of a Single Mode Fiber (SMF) and an in-line amplifier (SOA) for the reach extension purpose, with an injected bias current $I_{\text {bias }}$. At the receiver side (just before wireless transmission), a photo-detector converts the optical power into an electrical voltage signal to be eventually attenuated before entering the antenna (so as to meet FCC mask). The same ADS software-based model as in [20] is adopted for the SOA (INPHENIX-IPSAD1501), as it has proved to be highly accurate and in very good agreement with experimental data. Increasing the transmission distance requires operating in the saturated region of the 


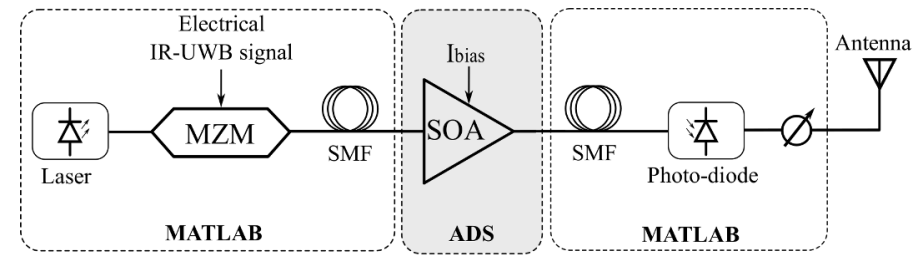

Fig. 1: Block diagram of the Impulse Radio over fiber system with Matlab/ADS co-simulation.

SOA, which yields various nonlinear impairments affecting the transmitted signal. On the non-coherent receiver side, the resulting distortion will be characterized in terms of power efficiency with respect to the FCC mask, defined as:

$$
\eta=\frac{\int_{3.1 \mathrm{GHz} z}^{10.6 \mathrm{GHz}} S_{p}(f) d f}{\int_{3.1 \mathrm{GHz}}^{10.6 \mathrm{GH} z} S_{F C C} d f} \times 100 \%
$$

where $S_{p}(f)$ denotes the power spectral density (PSD) for a pulse $p(t)$ [19]. $\eta$ is computed after photo-detector while considering antenna effects are ignored. To have a look at SOA impact, fig. 2 shows the spectral response of the 5-th order derivative Gaussian pulse in electrical domain (solidblue) and after being passed through SOA (dashed-green), without propagation in fiber. The PSD of the optical pulse violates FCC in the most restricted region corresponding to GPS band $[0.96-1.61] \mathrm{GHz}$, where the low power level $(-75.3 \mathrm{dBm} / \mathrm{MHz})$ is greatly challenging. So the average signal power has to be reduced so as to meet this limit, leading to a significant impact on the power efficiency (degradation $>30 \%$ ). Hence, spectrum enhancement requires eliminating the low frequency components induced by SOA. A simple criterion for evaluating this spectral impact corresponds to the ratio between the power collected in the $[0,2] \mathrm{GHz}$ band with respect to the overall PSD:

$$
P S R_{2 G H z}=\frac{\int_{0}^{2 G H z} S_{p}(f) d f}{\int_{0}^{\infty} S_{p}(f) d f} \times 100 \%
$$

This criterion is analyzed in fig. 3 for the case of the $5^{\text {th }}$ derivative Gaussian pulse, with different peak-to-peak voltage and laser power, where $I_{\text {bias }}=250 \mathrm{~mA}$. A quick rise of the PSR can be clearly observed, especially for $V_{p p}>0.5 V_{\pi}$ where strong nonlinearities act on the pulse waveform. In the following, we investigate the benefits of electrical pulse precompensation with the view to avoid any power efficiency degradation. The power efficiency of the $5^{t h}$ order Gaussian pulse is plotted in fig. 4 versus SOA input power, for $I_{\text {bias }}=$ $250 \mathrm{~mA}$. When the Amplified Spontaneous Emission (ASE) noise is ignored, a degradation in the power efficiency is observed as we move towards higher input powers. The bad impact of SOA particularly occurs at large $V_{p p}$ (e.g. $V_{p p}=$ $0.75 V \pi)$, where the drop in the efficiency results from the large spectral distortion. On the opposite, for $V_{p p}=0.25 V \pi$, a semi-linear gain behavior is ensured, leading to a very good spectral efficiency (approximately 50\%) over a broad range of input power, reaching the saturation region $(0 \mathrm{dBm})$. In

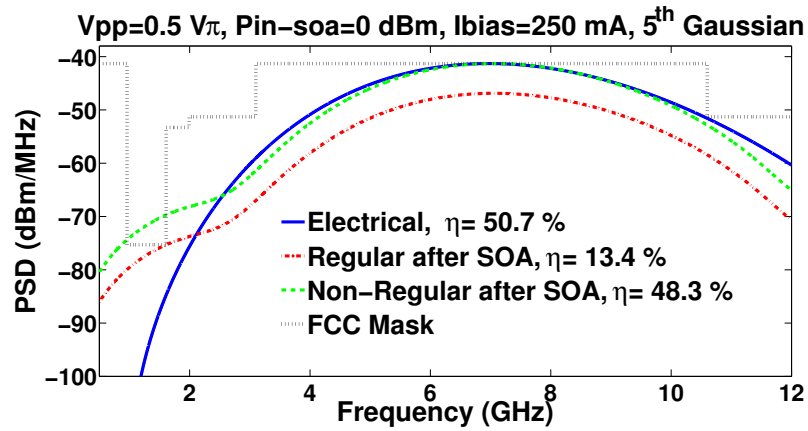

Fig. 2: Power Spectral Density of $5^{\text {th }}$ Gaussian in electrical domain and after SOA (regular and irregular with FCC).

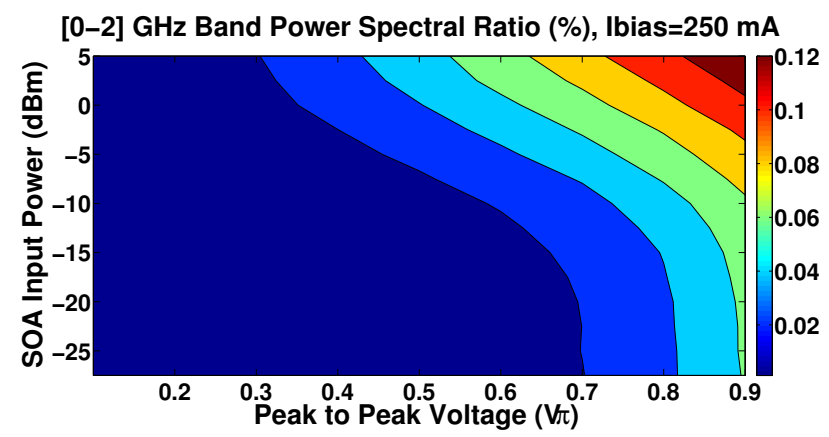

Fig. 3: $P S R_{2 G H z}$ of $5^{\text {th }}$ Gaussian versus SOA input power and peak-to-peak voltage, where no fiber is considered.

the noisy case, the power efficiency is evaluated via MonteCarlo simulations with the computation of the average overs 100 runs; as can be clearly seen, the pseudo-linear gain characteristics is no longer preserved due to the larger noise influence at lower input power. Thus, there is a compromise between linearity and optical signal to noise ratio (OSNR), which depends upon the peak-to-peak voltage. As can be seen, the ASE impact on pulse dynamics tends to be reduced for large $V_{p p}$; it is a positive aspect but at the cost of nonlinearities. For $V_{p p}=0.25 V \pi$, the rise in efficiency is due to the increasing OSNR, combined with limited nonlinear effects. Prior to strong saturation, a slight increase in the efficiency $(<5 \%)$ is noticed for the noisy signals over normal ones, for any $V_{p p}$, that is due to the power added by ASE noise within the UWB mask. We can systematically verify the convergence between solid and dashed lines at very high input power, resulting from a negligible noise power. For the remaining part of this paper, we will assume a medium value $V_{p p}=0.5 \mathrm{~V} \pi$, which is interesting in the sense that it both represents the noisy and nonlinear characteristics typically associated with SOA.

\section{SOA NONLINEARITIES Mitigation VIA ELECTRICAL PULSES PRE-DistorTiON}

A broad variety of linearization techniques are discussed in the literature [16]-[18], [21] for reducing the nonlinear effects associated to optical communication systems. The different approaches can be classified into two main groups, electrical 


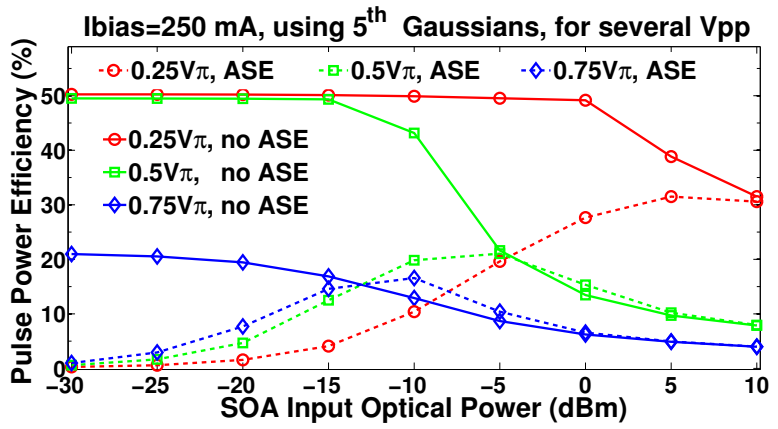

Fig. 4: Power Efficiency of $5^{\text {th }}$ Gaussian versus SOA input power for several peak-to-peak voltages, where no fiber is considered.

linearization and optical linearization. Optical schemes can operate over a wide bandwidth and some techniques are even specifically designed for SOA [22], [23], but due to either high cost or bandwidth limitations associated to radio frequency control circuits, these schemes are not considered in our study. Regarding the methods operating in the electrical domain, digital linearization (pre-distortion, post-distortion) is interesting for its flexibility but we see the bandwidth/sampling requirements as a drawback for a low cost IR-UWB system; so, we preferred to investigate the alternative of analog predistortion, consisting in designing circuits at the transmitter side so as to suppress the nonlinear distortion components resulting from SOA amplification. As reported in various studies, tunable UWB pulse generators can be designed at low cost in CMOS technology [24]-[26]. Considering such

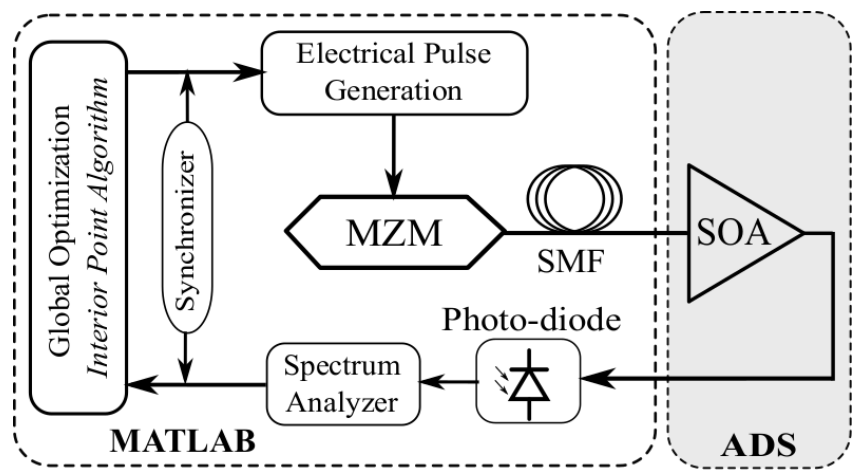

Fig. 5: Block diagram for the optimization process used for designing pre-distorted electrical waveforms.

techniques, we made the assumption of a tunable Gaussian pulse generator, so that the generated pulse $x(t, \mathbf{p})$ can be shaped with arbitrary parameters $\mathbf{p}$ : shaping factor for the case of a single Gaussian pulse, or pulse shaping factors, weighting coefficients, besides to the delay for a linear combination of Gaussian pulses. Then our aim is to identify the best tuning parameters $\mathbf{p}^{*}$ in the sense of a maximized power efficiency at the SOA output. In this study, we solve this global optimization problem via an interior point algorithm [27], using a numerical model of the component, as illustrated in fig. 5. All distinct parameters are initialized at their optimum in electrical domain, so the algorithm can start from these values to re-optimize the pulse shape obtained at SOA output. Note that our approach presents some similarities with a work of Mirshafiei et al. [28], who addressed the pre-compensation against antenna effects, based on combinations of Gaussian pulses generated in the optical domain. Our contribution, whose a first investigation has been reported in [29], is different in the sense that we aim at mitigating SOA nonlinearities, which are very specific, and we consider that the pulses are generated/pre-distorted in the electrical domain. Regarding the practical aspect, this process could be automatically implemented in a closed loop transceiver system, utilizing software-to-hardware interfaces. In our setup, SOA was placed at $40 \mathrm{~km}$, the operating point corresponding to an input power $P_{S O A}=0 \mathrm{dBm}$, which is a strong gain saturation point, while MZM is driven by $V_{p p}=0.5 V \pi$. These operating conditions are fixed for the remaining part of the study.

\section{A. Case of 5-th derivative Gaussian pulse}

The high power efficiency offered by the $5^{t h}$ derivative Gaussian pulse, besides to the acceptable order of generation complexity, both make it an attractive target for impulse radio system designers [30]. As shown in (3), only one variable could be calibrated in this waveform, which is the pulse shaping factor $(\sigma)$. Such a parameter has a simultaneous influence on the $10 \mathrm{~dB}$ bandwidth $\left(B_{10}\right)$ and on the central frequency. Figure 6 describes the original and pre-distorted (PD) pulses in electrical domain and at SOA output, in the case of placing the photo-detector directly after SOA, where the spectra are plotted in the noise-free case (ASE noise not considered for spectrum readability). We can clearly see that the pre-distorted pulse exhibits only a small improvement in power efficiency $(<5 \%)$, this is due to the single degree of freedom. Therefore it is better to search for more appropriate pulse shapes to be adopted with pre-distortion.
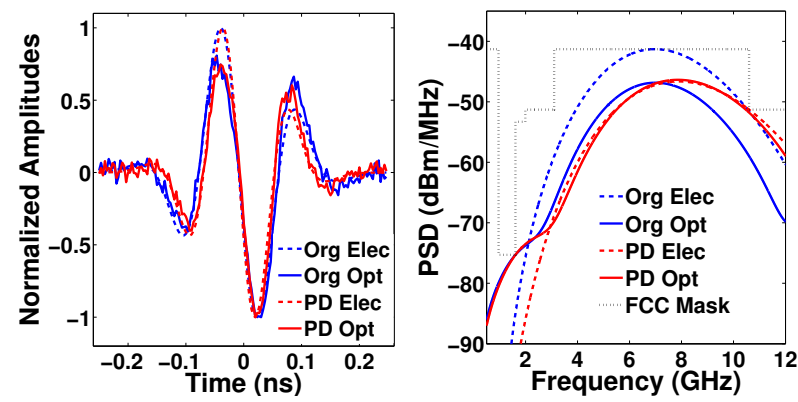

Fig. 6: Original (Org) and pre-distorted (PD) $5^{\text {th }}$ Gaussian, in electrical domain (Elec) and after SOA (Opt).

$$
x_{5}(t)=\frac{A}{\sqrt{2 \pi}}\left(\frac{-t^{5}}{\sigma^{11}}+\frac{10 t^{3}}{\sigma^{9}}-\frac{15 t}{\sigma^{7}}\right) \exp \left(-\frac{t^{2}}{2 \sigma^{2}}\right)
$$

\section{B. Higher order of Gaussian derivative}

In reality, to avoid hitting the GPS band, the spectrum must be shifted towards higher frequencies. The spectral peak for 


\begin{tabular}{|c|c|c|c|c|c|c|c|c|c|c|}
\hline Pulses & $\sigma_{1}(\mathrm{ps})$ & $\sigma_{2}(\mathrm{ps})$ & $\sigma_{3}(\mathrm{ps})$ & $a_{2} / a_{1}$ & $a_{3} / a_{1}$ & $\eta(\%)$ Elec & $f_{\text {peak }}(\mathrm{GHz})$ & $B_{10}(\mathrm{GHz})$ & $B L_{34}(\mathrm{GHz})$ & $\eta(\%) \mathrm{SOA}$ \\
\hline $\begin{array}{c}\text { Original } 5^{t h} \\
\text { Gaussian }\end{array}$ & 51 & $\mathrm{x}$ & $\mathrm{x}$ & $\mathrm{x}$ & $\mathrm{x}$ & 50.7 & 7 & 6.17 & 6.11 & 13.7 \\
\hline $\begin{array}{l}\text { Pre-distorted } \\
5^{\text {th }} \text { Gaussian }\end{array}$ & 45.07 & $\mathrm{x}$ & $\mathrm{x}$ & $\mathrm{x}$ & $\mathrm{x}$ & 15.74 & 7.86 & 6.95 & 6.88 & 17.2 \\
\hline $\begin{array}{l}\text { Original } 6^{t h} \\
\text { Gaussian }\end{array}$ & 53.83 & $\mathrm{x}$ & $\mathrm{x}$ & $\mathrm{x}$ & $\mathrm{x}$ & 47.94 & 7.23 & 6.13 & 6.41 & 17 \\
\hline $\begin{array}{l}\text { Pre-distorted } \\
6^{\text {th }} \text { Gaussian }\end{array}$ & 72.45 & $\mathrm{x}$ & $\mathrm{x}$ & $\mathrm{x}$ & $\mathrm{x}$ & 37.28 & 5.5 & 4.85 & 3.92 & 38 \\
\hline $\begin{array}{c}\text { Original } \\
x_{C_{D}}\end{array}$ & 43.02 & 43.02 & $\mathrm{x}$ & -1 & $\mathrm{x}$ & 57.2 & 6.45 & 6.84 & 6.05 & 6.96 \\
\hline $\begin{array}{c}\text { Pre-distorted } \\
x_{C_{D}} \\
\end{array}$ & 61.68 & 40.47 & $\mathrm{x}$ & -1.24 & $\mathrm{x}$ & 1.6 & 6.2 & 7.34 & 4.56 & 54.1 \\
\hline $\begin{array}{c}\text { Original } \\
x_{C_{G}}\end{array}$ & 34.27 & 83.96 & 49.98 & 0.12 & -0.89 & 56.47 & 6.13 & 6.74 & 3.81 & 7.1 \\
\hline $\begin{array}{c}\text { Pre-distorted } \\
x_{C_{G}}\end{array}$ & 80.71 & 60.71 & 28.03 & 2.81 & -3.3 & 1.53 & 6.1 & 7.45 & 4.35 & 56.1 \\
\hline
\end{tabular}

TABLE I: Parameters $\left(\sigma_{1}, \sigma_{2}, \sigma_{3}, a_{2} / a_{1}, a_{3} / a_{1}\right)$ of electrical generated pulses, and the spectral characteristics $\left(f_{\text {peak }}, B_{10}, B L_{34}\right)$ of corresponding waveforms at SOA output (placed at $40 \mathrm{~km}$ ). Besides to the power efficiencies $(\%)$ in electrical domain and after SOA, with $P_{S O A}=0 \mathrm{dBm}, I_{\text {bias }}=250 \mathrm{~mA}$, and $V_{p p}=0.5 V_{\pi}$.

any $n^{\text {th }}$ order Gaussian pulse derivative $x_{n}(t)$ is $f_{\text {peak }}=\frac{\sqrt{n}}{2 \pi \sigma}$, where the spectrum is expressed as

$$
X_{n}(f)=A(j 2 \pi f)^{n} \exp \left(-\frac{(2 \pi f \sigma)^{2}}{2}\right)
$$

To step up the power spectral density under FCC, we have to reduce $B_{10}$ by utilizing higher values of $\sigma$, as illustrated in (4). Increasing $\sigma$ can be compensated by augmenting $n$, hence making a separate control for $B_{10}$ and $f_{\text {peak }}$. Adopting a higher order of derivation gains more flexibility in shaping the spectrum, as the latter being far from the most severely powerrestricted band (corresponding to GPS), and consequently the design of a pre-distorted waveform becomes much easier. The benefit of the 6-th derivative Gaussian appears in fig. 7, with a time domain expression given in (5). By lowering the 10 $\mathrm{dB}$ bandwidth of the pre-distorted pulse spectrum, we have enabled an increase in the efficiency (> 20\%) without violating the regular limit. In this case, the pre-distortion block could be implemented as a simple differentiator, which can enhance the performance at limited complexity.

$$
x_{6}(t)=\frac{A}{\sqrt{2 \pi}}\left(-\frac{15}{\sigma^{7}}+\frac{45 t^{2}}{\sigma^{9}}-\frac{15 t^{4}}{\sigma^{11}}+\frac{t^{6}}{\sigma^{13}}\right) \exp \left(-\frac{t^{2}}{2 \sigma^{2}}\right)
$$

\section{Abraha's Combination of Gaussian Doublets}

Good results have been achieved with single pulse predistortion; unfortunately, the number of adjustable parameters limits the improvement in the spectral efficiency. A more effective technique is to apply a combination of waveforms, in order to get a superior control of the resultant pulse dynamics, as will be presented in the sequel. Some combinations have been proposed in the literature [31] [32], but taking into account practical constraints, it is better to conserve simple combinations within the Gaussian family. We have considered the work of Abraha et al., who have recently developed highly effective pulse shapes based on either monocycles or
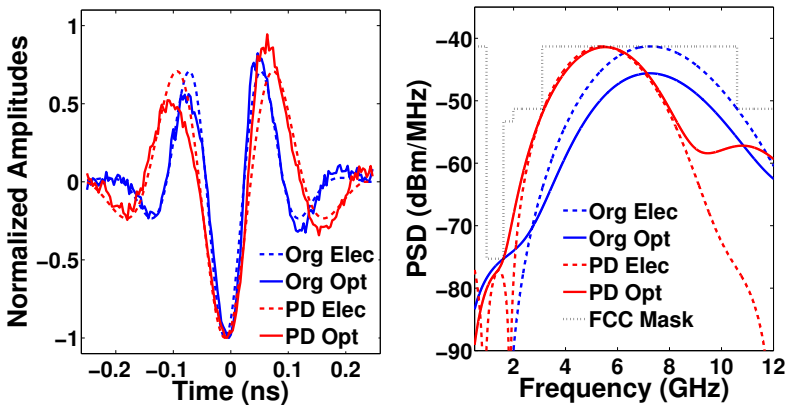

Fig. 7: Original (Org) and pre-distorted (PD) $6^{\text {th }}$ Gaussian, in electrical domain (Elec) and after SOA (Opt).

doublets [19]. As the latter approach relies on higher order of Gaussian derivative, with a better spectral efficiency, it will be the candidate in our work. Hence, two basic Doublets $x_{21}(t), x_{22}(t)$ will be considered, having different weighting coefficients and shaping factors:

$$
\begin{gathered}
C_{D}(t)=a_{1} x_{21}(t)+a_{2} x_{22}(t-\tau) \\
x_{2 i}(t)=\frac{A}{\sqrt{2 \pi} \sigma_{i}^{3}}\left(k \frac{t^{2}}{\sigma_{i}^{2}}-1\right) \exp \left(-\frac{t^{2}}{2 \sigma_{i}^{2}}\right)
\end{gathered}
$$

$a_{1}$ and $a_{2}$ are the weighting factors, $\tau$ is a time delay between pulses, fixed at $\tau=25 \mathrm{ps}$, and $k=1.16$ is an arbitrary scaling of the modified Doublet. Table I displays all the parameters of utilized pulses, besides to the power efficiencies in electrical domain and at SOA output (the spectral characteristics being evaluated at this point). $B L_{34}$ is defined as the left -34 $\mathrm{dB}$ bandwidth (frequency space between $f_{L}$ and $f_{\text {peak }}$ ), this criteria is useful to evaluate the spectrum in correspondence with GPS band. The term $A$ is always set at 1 , as it has no influence on time dynamics for single waveforms; and in case of combinations, only the relative amplitude between basic pulses is important, since the real intensity for signals at photodetector output is controlled by the laser power and peak- 

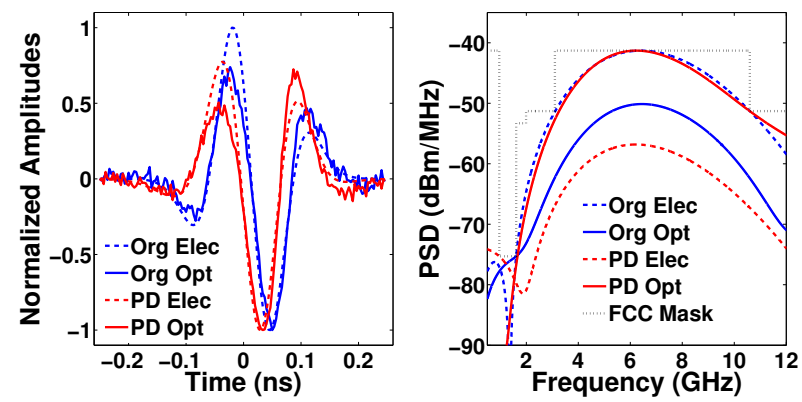

Fig. 8: Original (Org) and pre-distorted (PD) $C_{D o u b}$, in electrical domain (Elec) and after SOA (Opt).

to-peak voltage. In Abraha's work, the doublets are similar with opposite polarities, as a high pass filtering is made via spectral oscillating term resulting from time delay. We don't preserve these conditions with pre-distortion, as the target is to mitigate SOA non-linearity by exploiting all the degrees of freedom associated to the linear combination of pulses. Hence, different shaping factors and weighting coefficients have been considered in our work. A significant jump in the power efficiency $(>45 \%)$ is illustrated in fig. 8 for the predistorted combination of Doublets. The increase in the 10 $\mathrm{dB}$ bandwidth from $6.84 \mathrm{GHz}$ to $7.34 \mathrm{GHz}$ has enlarged the spectral coverage area under FCC mask, besides to the 1.5 $\mathrm{GHz}$ reduction in $B L_{34}$, which permits a step up in the PSD without exceeding GPS limit. The peak of the spectrum exactly matches FCC limit $(-41.3 \mathrm{dBm} / \mathrm{MHz})$ at $f_{\text {peak }}=6.2 \mathrm{GHz}$, which is not so far from the center of the UWB mask, thanks to pre-distortion.

\section{A new waveform based on a combination of fundamental Gaussians}

Increasing the number of tuning parameters in a waveform has shown to be more pertinent for pre-distortion. A new pulse shape is now proposed, based on a linear combination of three fundamental Gaussian pulses:

$$
C_{G}(t)=a_{1} g_{1}(t)+a_{2} g_{2}(t)+a_{3} g_{3}(t)
$$

where

$$
g_{i}(t)=\exp \left(-\frac{t^{2}}{2 \sigma_{i}^{2}}\right)
$$

Such a combination enables a pre-distortion with 5 variables $\left(\sigma_{1}, \sigma_{2}, \sigma_{3}, a_{2} / a_{1}, a_{3} / a_{1}\right)$, which promises for a better power efficiency obtained at SOA output. The weighting coefficients have been optimized as described in table I. The resultant waveform is depicted in fig. 9 (left); it has a negligible DC component due to the opposite polarities of Gaussian amplitudes, with an energy being concentrated at a higher frequency range due to zero crossings. The peak frequency is $6.14 \mathrm{GHz}$, where the $3 \mathrm{~dB}$ and $10 \mathrm{~dB}$ bandwidths are $B_{3}=4.1 \mathrm{GHz}$ and $B_{10}=7.5 \mathrm{GHz}$ respectively, which is in very good agreement with the UWB spectral mask. As illustrated in fig. 9 (right), a highly efficient pulse waveform is obtained, offering a power efficiency of $\eta=56.47 \%$, which outperforms the 5 -th derivative
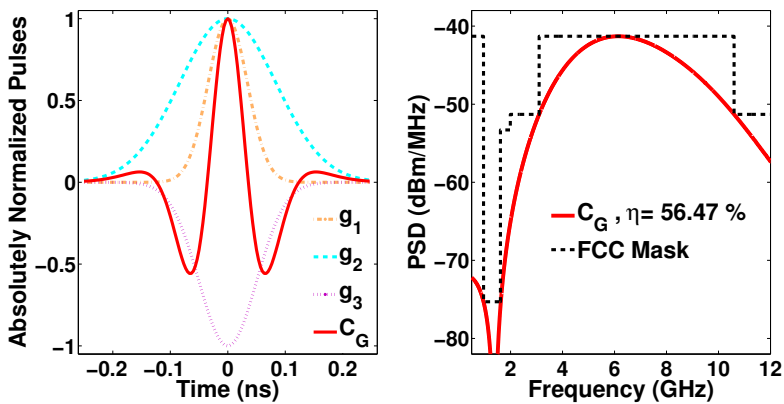

Fig. 9: Our proposed combination $C_{G}$ based on the pulses $g_{i}$, in time (left) and frequency (right) domain.

Gaussian pulse (50.7\%) and is close to Abraha's combination of Doublets (57.2\%) in electrical domain. An important feature associated with our approach is the absence of any time differentiator nor delay line, which reduces the system cost and complexity [19], [30], [33]. As can be seen in fig. 10, our combination has carried out the best power efficiency at SOA output $(\eta=56.1 \%)$, which performs better than Abraha's combination of Doublets (54.1\%), thanks to the larger number of tuning parameters. After SOA, the pre-distorted spectrum has a close peak frequency to the original one $(6.1 \mathrm{GHz})$, but covering a larger $10 \mathrm{~dB}$ bandwidth $(7.45 \mathrm{GHz})$. Here there is a special case, the original spectrum at SOA output hits FCC mask at low frequencies due to the presence of a significant left side lobe, the main lobe staying far from the FCC limit even at the $34 \mathrm{~dB}$ band. This spectral characteristic results from the combining of time spread and windowing effect, which can be efficiently overcome by designing a predistorted pulse (better distribution of the pulse energy inside the time window). Unfortunately, the pulse undergoes a time
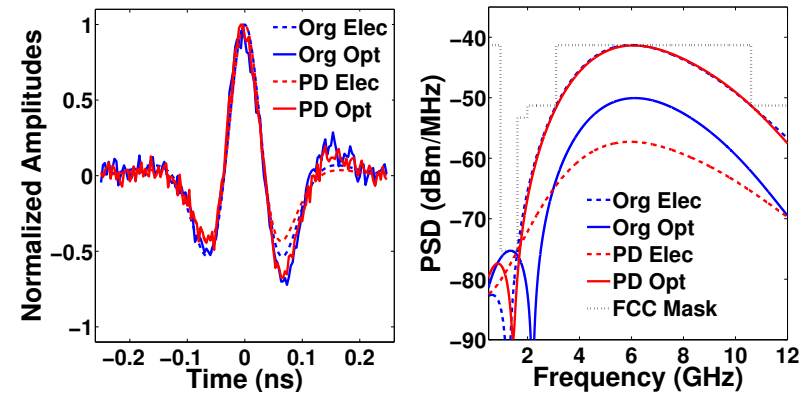

Fig. 10: Original (Org) and pre-distorted (PD) $C_{G}$, in electrical domain (Elec) and after SOA (Opt).

spread after amplification, thus the energy outside the $0.5 \mathrm{~ns}$ slot is no more negligible, which translates into significant sidelobes in frequency domain (sinc influence). The predistortion processing has enabled a significant change in the pulse dynamics so as to render this time windowing effect negligible. 


\section{E. Bias current influence}

Efficient pulses in electrical domain are usually more affected by the optical system non-linearity. We can see in fig. 11 that for low biasing currents $(100 \mathrm{~mA})$, the $5^{\text {th }}$ and $6^{\text {th }}$ Gaussian pulses have a small degradation in the power efficiency; whereas a significant decrease $(>30 \%)$ is noticed for the combination of Doublets. Applying a larger biasing current increases the order of nonlinearity, which leads to lower spectral efficiencies for all pulses. Nevertheless, we observe that no great change occurs after $200 \mathrm{~mA}$, due to the optical gain saturation at $0 \mathrm{dBm}$ input power. Pre-distortion could be applied with all biasing currents, but with the view to achieve a reach extension the value of $250 \mathrm{~mA}$ is adopted, as associated with the highest optical power at SOA output. For such conditions, our pre-distorted pulse offers a neat advantage over the 5-th or 6-th derivative Gaussian waveforms and is slightly more effective than Abraha's pre-distorted pulse.

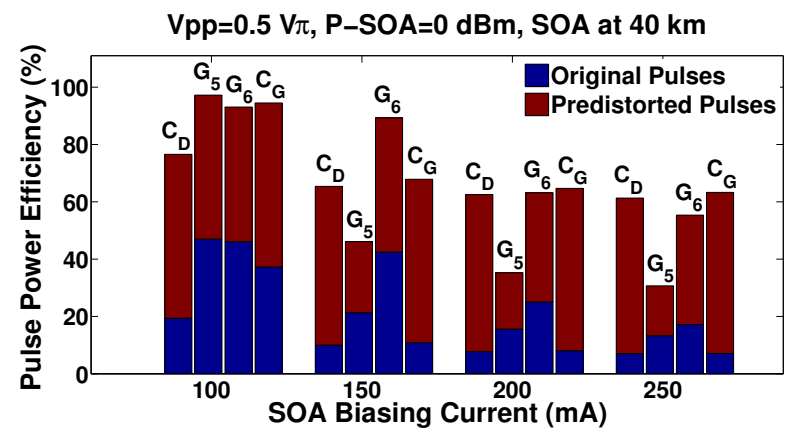

Fig. 11: Power Efficiency of original and pre-distorted pulses directly after SOA (at $40 \mathrm{~km}$ ) for several biasing currents.

F. Robustness against uncertainty for single waveforms and combinations

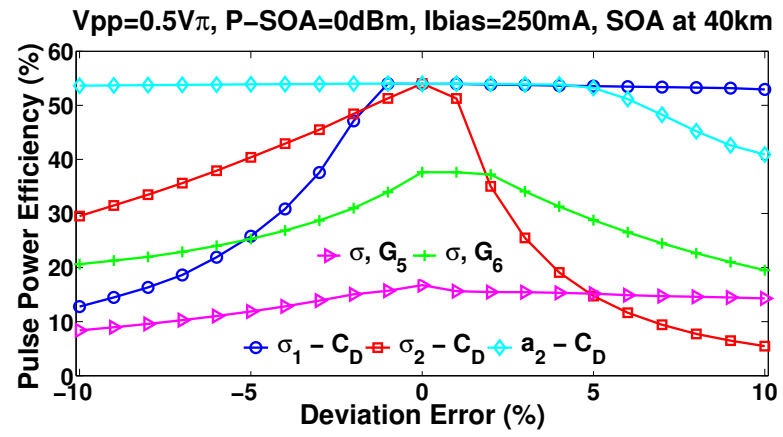

Fig. 12: Robustness against uncertainty for the $5^{t h}$ and $6^{t h}$ Gaussian pulses, besides to Abraha's combination of Doublets.

Until now only theoretical models have been considered for the different pulse shapes. But from a practical point of view, some uncertainties are expected to occur in the CMOS circuits that could be designed to generate the various pulses. So, we investigate in this sub-section the impact of any parametric deviation with respect to the optimum solution discussed previously. Figure 12 illustrates how the power

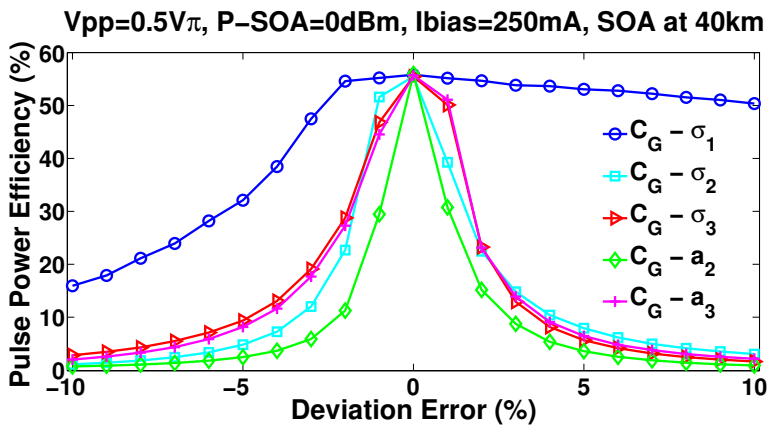

Fig. 13: Robustness against uncertainty for our proposed pulse based on fundamental Gaussians.

efficiency is affected by a parametric uncertainty for the 5 -th and 6-th Gaussian pulses, together with the Doublets combination. $G_{5}$ and $G_{6}$ show a kind of immunity against systematical errors, as $\sigma$ is the unique variable which controls the dynamics of a single pulse. The problem appears with Abraha's combination, which exhibits a relative robustness against $\sigma_{1}$ and $a_{2}$ deviations; unfortunately, any slight change in $\sigma_{2}$ is enough to drop off the spectral efficiency, specially for negative deviation errors, knowing that it is not a simultaneous change in the combination variables. We can figure out that the newly designed pulse is the most sensitive against system uncertainties, as illustrated in fig. 13. In fact, it is much more influenced than the other waveforms, as it relies on a larger number of pulses, and all parameters have been optimized simultaneously, so any change in one of the variables makes all the set no more optimal. Besides, pure Gaussian pulses have strong DC component, which can re-appear in our combination due to any lose in the basic pulses symmetry (positive/negative symmetry which cancels the DC), and consequently lead to a fast drop in the power efficiency $(\eta<5 \%$ for a deviation error of $10 \%)$.

\section{Case of a Modulated Impulse Radio Signal PROPAGATING OVER OpticAl FibER LINK}

In our simulations, we take into account the effects of the optical link (attenuation and chromatic dispersion) before SOA, as they have an influence on the pulse dynamics entering the amplifier, depending on the covered distance, fiber input power, and peak-to-peak power swing. Therefore, the optimum parameters of pre-distorted waveforms have been extracted considering SOA located at $40 \mathrm{~km}$, where the operating conditions are the same as mentioned in table I. In order to evaluate our pre-distortion scheme considering a stream of modulated pulses, a new criterion of power efficiency has been defined as

$$
\eta_{M}=\frac{P_{\mathcal{F}}}{\max \left(\overline{P_{\mathcal{F}}}\right)}
$$

where $P_{\mathcal{F}}$ stands for the power collected over a frequency band of interest $\mathcal{F}$ (typically the [3.1-10.6] $\mathrm{GHz}$ band) for the electrical signal at antenna input, and $\max \left(\overline{P_{\mathcal{F}}}\right)$ denoting the total power evaluated over the same band for an OOK 
or PPM modulated signal based on the sinc pulse, which is optimal in the sense that it corresponds to a $100 \%$ spectrum use (full coverage of the spectral mask). The advantage of pre-distortion is depicted in fig. 14 for a $1.5 \mathrm{Gbps}$ OOK, so we can gain an improvement over a large enough traveling distance $(40 \mathrm{~km}$ to $150 \mathrm{~km})$, knowing that no pre-amplifier has been placed for modulated signals before the antenna. The degradation in the curves is due to frequency shaping and deviation caused in fiber [9] (with a chromatic dispersion of $17 \mathrm{ps} / \mathrm{nm} / \mathrm{km}$ ), so the central frequency and $B_{10}$ are no more preserved specially at large extension link. This improvement is justified by the fact that, the envelope of spectral spikes for OOK or PPM takes the shape of basic transmitted waveform, so perfect pulse shapes lead to an efficient spectrum associated with the modulated impulse radio signal. Between $40 \mathrm{~km}$ and $100 \mathrm{~km}$, we can see a

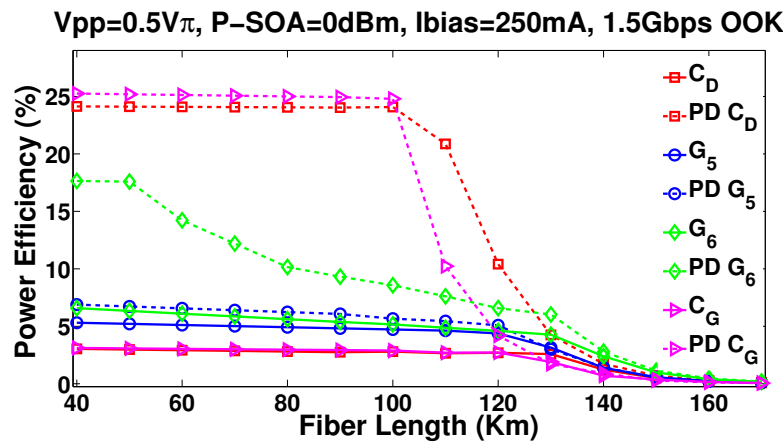

Fig. 14: Power Efficiency versus fiber length for $1.5 \mathrm{Gbps}$ OOK based on the utilized pulses, P-SOA $=0 \mathrm{dBm}, I_{\text {bias }}=250 \mathrm{~mA}$, $V_{p p}=0.5 \mathrm{~V} \pi$, where SOA is placed at $40 \mathrm{~km}$.
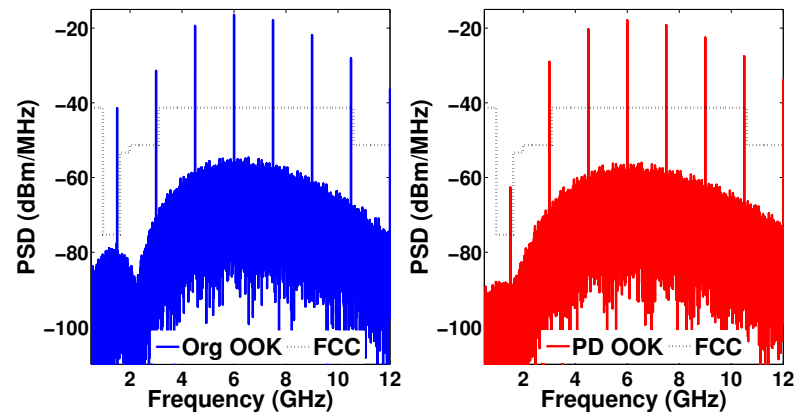

Fig. 15: Irregular PSD of $1.5 \mathrm{Gbps}$ OOK based on original (left) and pre-distorted (right) $C_{G}(t)$ after SOA (at $40 \mathrm{~km}$ ), where $\mathrm{P}-\mathrm{SOA}=0 \mathrm{dBm}, I_{\text {bias }}=250 \mathrm{~mA}, V_{p p}=0.5 \mathrm{~V} \pi$.

flat response in the curves corresponding to combinations of pulses, which results from the fact that all spectra are already above FCC limit, thus the signal has to be systematically attenuated so as to respect the mask. Our waveform exhibits a faster degradation than Doublets at large distances, as it has less immunity against fiber effects. Regarding practical aspects, we can say that power efficiency enhancement comes at the price of a larger complexity; depending on application constraints, adopting higher order of derivation with a
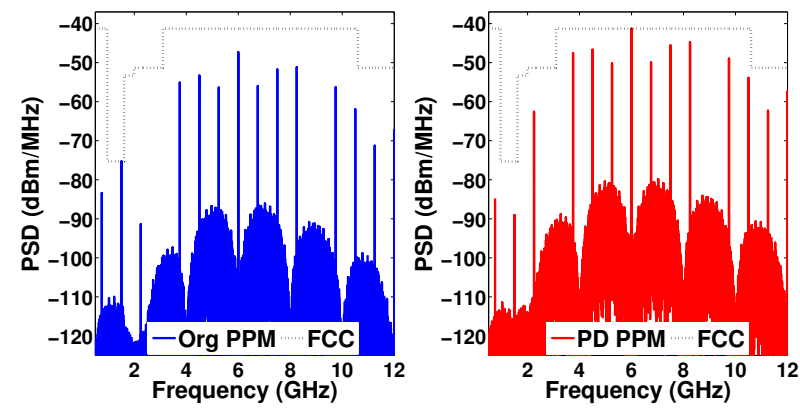

Fig. 16: Regular PSD of 0.75 Gbps PPM based on original (left) and pre-distorted (right) $C_{G}(t)$ after SOA (at $40 \mathrm{~km}$ ), where $\mathrm{P}-\mathrm{SOA}=0 \mathrm{dBm}, I_{\text {bias }}=250 \mathrm{~mA}, V_{p p}=0.5 V \pi$.

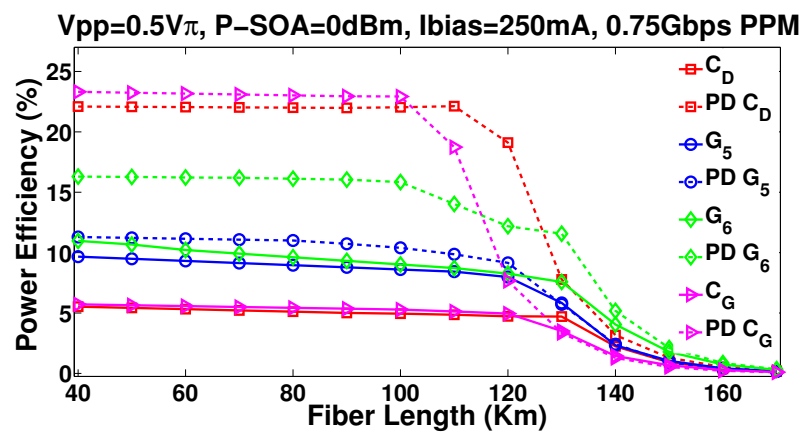

Fig. 17: Power Efficiency versus fiber length for 0.75 Gbps PPM based on the utilized pulses, $\mathrm{P}-\mathrm{SOA}=0 \mathrm{dBm}, I_{\text {bias }}=250 \mathrm{~mA}$, $V_{p p}=0.5 \mathrm{~V} \pi$, where $\mathrm{SOA}$ is placed at $40 \mathrm{~km}$.

single pulse shape could be more suitable than applying a combination of pulses, and utilizing a combination of two pulses may be much simpler than using a set of three pulses. The PSD of the $C_{G}$ - based OOK signal at SOA output is plotted in fig. 15, with no attenuation. Apparently, the spectral shaping at low frequency band is the reason beyond efficiency enhancement, so a higher power level can be achieved by the spectra when adapted to FCC mask, as shown in fig. 16 for a 0.75 Gbps PPM. Figure 17 proves that the same conclusion could be drawn for PPM regardless the covered distance in the optical link, where PPM index is $0.5 \mathrm{~ns}$. Therefore, the proposed pre-distortion technique has always validated a good potential, while reaching a coverage of $150 \mathrm{~km}$, fitting the request of Fiber-to-the-Home (FTTH) networks reported in the literature [34]. The improvement in the power efficiency translates into a higher signal-to-noise ratio obtained at the antenna output, which promises with a better bit error rate performance in energy detection receivers.

\section{CONCLUSION}

A low cost SOA in-line amplifier has been considered in this study for the purpose of reach extension of IR-UWB over fiber systems. Based on a realistic SOA physical model, analog pre-distortion schemes have been investigated for coping with SOA nonlinearities and with the view to improve power efficiency. Two linear combinations of Gaussian pulses 
were utilized, the first is based on Abraha's combination of Doublets, and the second has been newly designed based on pure Gaussians. Both have proved to be highly effective for correcting SOA impact. Using higher orders of Gaussian derivatives is an alternative solution offering a lower potential but with less complexity. Over a large extension of the optical link, our pre-distortion approach has exhibited an enhancement in the power efficiency of $1.5 \mathrm{Gbps}-\mathrm{OOK}$ and $0.75 \mathrm{Gbps}-$ PPM. Experiments will be conducted in our future work so as to validate the proposed schemes, while considering bit error rate analysis and including antenna effects.

\section{REFERENCES}

[1] M. Ghavami, L. Michael, and R. Kohno, "Ultra wideband signals and systems in communication engineering," John Wiley \& Sons, 2007.

[2] G. R. Aiello and G. D. Rogerson, "Ultra-wideband wireless systems," IEEE Microwave Magazine, 4(2), pp. 36-47, 2003.

[3] I. Oppermann, M. Hämäläinen, and J. Iinatti, "UWB: theory and applications," John Wiley \& Sons, 2005.

[4] FCC Report and Order. In the Matter of Revision of Part 15 of the Commission's Rules Regarding Ultra-Wideband Transmission Systems. FCC 02-48, Apr. 2002.

[5] M. Z. Win and R. A. Scholtz, "Impulse radio: How it works," IEEE Communications Lett., 2(2), pp. 36-38, 1998.

[6] J. R. Fernandes and D. Wentzloff, "Recent advances in IR-UWB transceivers: An overview," In Circuits and Systems (ISCAS), Proceedings of 2010 IEEE International Symposium, pp. 3284-3287, 2010.

[7] C. Lim, A. Nirmalathas, M. Bakaul, P. Gamage, K. L. Lee, Y. Yang, R. Waterhouse, "Fiber-wireless networks and subsystem technologies," IEEE J. Lightw. Technol., 28(4), pp. 390-405, 2010.

[8] F. Zeng, and J. Yao, "An approach to ultrawideband pulse generation and distribution over optical fiber," IEEE Photon. Technol. Letters, 18(7), pp. 823-825, 2006.

[9] S. Pan, and J. Yao, "Performance evaluation of UWB signal transmission over optical fiber," IEEE J. Selected Areas in Communications, vol. 28, no.6, pp. 889-900, 2010.

[10] M. Popov, "The convergence of wired and wireless services delivery in access and home networks," In Optical Fiber Communication Conference (p. OWQ6). Optical Society of America, 2010.

[11] N. J. Gomes, M. Morant, A. Alphones, B. Cabon, J.E. Mitchell, C. Lethien, and S. Iezekiel, "Radio-over-fiber transport for the support of wireless broadband services," J. of Optical Networking, 8(2), pp. 156$178,2009$.

[12] F. Zeng, Q. Wang, and J. Yao, "All-optical UWB impulse generation based on cross-phase modulation and frequency discrimination," Electron. Lett., 43(2), pp. 119-121, 2007.

[13] M. Bolea, J. Mora, B. Ortega, and J. Capmany, "Optical UWB pulse generator using an $\mathrm{N}$ tap microwave photonic filter and phase inversion adaptable to different pulse modulation formats," Optics express, 17(7), pp. 5023-5032, 2009.

[14] Y. Yu, J. Dong, X. Li, and X. Zhang, "Ultra-wideband generation based on cascaded Mach-Zehnder modulators," IEEE Photon. Technol. Letters, 23(23), pp. 1754-1756, 2011.

[15] M. J. Connelly, Semiconductor Optical Amplifiers. Boston, MA:Kluwer, 2002.

[16] K. Roberts, L. Chuandong, L. Strawczynski, M. O'sullivan, and I. Hardcastle, "Electronic precompensation of optical nonlinearity," IEEE Photon. Technol. Letters, 18(1-4), 403-405, 2006.

[17] E. Ip and J. M. Kahn, "Compensation of dispersion and nonlinear impairments using digital back propagation," J. Lightw. Technol., 26(20), $3416-3425,2008$.

[18] A. J. Lowery, "Fiber nonlinearity pre-and post-compensation for longhaul optical links using OFDM," Optics Express, 15(20), 12965-12970, 2007.

[19] S.T. Abraha, C. Okonkwo, P. A. Gamage, E. Tangdiongga, and T. Koonen, "Routing of power efficient IR-UWB wireless and wired services for in-building network applications," J. Lightw. Technol., 30(11), pp. 1651-1663, 2012.
[20] H. Khaleghi, P. Morel, A. Sharaiha, and T. Rampone, "Experimental validation of numerical simulations and performance analysis of a coherent optical-OFDM transmission system employing a semiconductor optical amplifier," J. Lightw. Technol., 31(1), pp. 161-170, 2013.

[21] X. Zhang, R. Zhu, D. Shen, and T Liu, "Linearization Technologies for Broadband Radio-Over-Fiber Transmission Systems," Photonics, vol. 1, no.4, pp. 455-472, 2014.

[22] C. Tai, S.-L. Tzeng, H.-C. Chang, and W. I. Way, "Reduction of nonlinear distortion in mqw semiconductor optical amplifier using light injection and its application in multichannel m-qam signal transmission systems," IEEE Photon. Technol. Lett. , vol. 10, pp. 609-611, Apr. 1998

[23] F. Tabatabai, H. S. Al-Raweshidy, "Feed-Forward Linearization Technique for Reducing Non-Linearity in Semiconductor Optical Amplifier," J. Lightw. Technol., vol.25, No. 9, pp.2667-2674, Sep. 2007.

[24] J. Han, C. Nguyen, "Ultra-wideband electronically tunable pulse generators", IEEE Microwave and Wireless Components Letters, vol. 14, No. 3, pp. $112-114,2004$.

[25] H. Kim, Y. Joo, and S. Jung, "A tunable CMOS UWB pulse generator," in Proc. IEEE International Conference on Ultra-Wideband, pp. 109-112, Waltham, MA, Sep. 24-27, 2006.

[26] R. Thai-Singama, F. Du-Burck, M. Piette, "Demonstration of a LowCost Ultrawideband Transmitter in the 3.1-10.6-GHz Band", IEEE Transaction on Circuits and Systems-II: Express briefs, vol. 59, no. 7, pp. 389-393, Jul. 2012.

[27] J. E. Mitchell, P. P. Pardalos, and M. G. C. Resende. Interior point methods for combinatorial optimization. In D.-Z. Du and P. Pardalos, editors, Handbook of Combinatorial Optimization , vol. 1, pp. 189-297. Kluwer Academic Publishers, 1998.

[28] M. Mirshafiei, M. Abtahi, and L. A. Rusch, "Ultra-wideband pulse shaping: bypassing the inherent limitations of the Gaussian monocycle," IET Communications, vol. 6, no. 9, pp. 1068-1074, 2012.

[29] H. Taki, S. Azou, A. Hamie, A. Al Housseini, A. Alaeddine and A. Sharaiha, "Pulse Shape Pre-distortion for Improving the Power Efficiency of SOA-based IR-UWB over Fiber Systems", 23rd IEEE Int. Conf. on Telecommunications (ICT 2016), 16-18 may 2016, Thessaloniki, Greece.

[30] H. Jin, L. Jiang, W. Hao, C. Sheng, H. Qijun, and Z. Yueping, "A CMOS fifth-derivative Gaussian pulse generator for UWB applications," Journal of Semiconductors, 35(9), 095005, 2014

[31] M. Wang, S. Yang, \& S. Wu, "A GA-based UWB pulse waveform design method. Digital Signal Processing," 18(1), 65-74, 2008.

[32] M. Matsuo, M. Kamada, \& H. Habuchi, "Design of UWB pulses based on B-splines," Circuits and Systems, ISCAS, IEEE International Symposium, pp. 5425-5428, 2005.

[33] H. Sheng, P. Orlik, A. M. Haimovich, Jr. L. J. Cimini, and J. Zhang, "On the spectral and power requirements for ultra-wideband transmission," In Communications, 2003. ICC'03. IEEE International Conference, Vol. 1, pp. 738-742, 2003.

[34] R. Llorente, T. Alves, M. Morant, M. Beltran, J. Perez, A. Cartaxo, and J. Marti, "Ultra-wideband radio signals distribution in FTTH networks," IEEE Photon. Technol. Lett., 20(11), pp. 945-947, 2008. 